\title{
Duct Ectasia an Unusual Case Presentation and an Overview
}

\author{
Dr. Anusha A.M., M.D \\ Assistant Professor, Department of Pathology, Dr PK DAS Institute of Medical Sciences, Palakkad, Kerala. \\ and Jubilee Centre for Medical Research, Thrissur, Kerala, India.
}

\begin{abstract}
Accessory breast is the presence of breast tissue at any part of the body except the normal breast region. Ectasia of the breast is a condition where the lactiferous ducts are blocked by secretions. Both accessory breast and duct ectasia are very common entities presenting separately but coexistence is rarity with a normal breast finding. In this case report, a 24-year-old female presented with bilateral painful axillary mass of 3 months duration which was excised is presented. Gross examination showed fibrofatty tissue with few areas of fibrosis while microscopic examination revealed breast tissue with duct ectasia. This case is reported for its rarity and to give clinicians a differential diagnosis of simultaneous coexistence of accessory breast with duct ectasia with a normal breast finding and a review of relevant aspects of duct ectasia is also included.
\end{abstract}

Keywords: Accessory breast, axilla, duct ectasia

\section{Overview}

Mammary duct ectasia is a benign condition characterized by dilatation of the mammary ducts [1]. Duct ectasia is an inflammatory condition of unknown genesis. Nipple retraction and discharge may be present, mimicking carcinoma clinically [2]. Mammary duct ectasia has also been referred to as varicocele tumor, comedomastitis, periductal mastitis, stale milk mastitis, chemical mastitis, granulomatous mastitis, or mastitis obliterans. Most of the cases probably represent a localized response to different components of stagnant colostrums [3]. It is found in $2-6 \%$ of women and $1-3 \%$ of males [4]. It occurs most often in postmenopausal women [5, 6] also being found to occur in younger women [5], children [7] and men [8].

Duct ectasia occur mostly in multiparous women in their $5^{\text {th }}$ or $6^{\text {th }}$ decade of life. In contrary to periductal mastitis, duct ectasia is not linked with cigarette smoking. The most common presentation is a palpable periareolar mass which is ill defined and frequently associated with nipple discharge and skin retraction. Pain and erythema are uncommon [9].

The mechanism of duct ectasia as described by Haagensen [1] begins with dilatation of the terminal collecting ducts beneath the nipple and areola. They become distended with cellular debris and lipoid containing material. The dilated ducts are bluish in colour and from $3-5 \mathrm{~mm}$ in diameter, at this stage there is no accompanying inflammation. The process ordinarily begins after menopause, although it is occasionally seen in younger women. The initial phase of duct ectasia is usually asymptomatic [9]. When symptomatic, the commonest clinical presentation is nipple discharge which is frequently bilateral coming through several ducts. This is yellowish or brownish but may finally become blood tinged. The discharge is spontaneous and intermittent [1].

As the disease progresses, the duct dilatation extends peripherally. At this stage histology reveals duct dilatation accompanied by inflammatory changes. The material distending the ducts irritates and causes thickening of the duct walls by fibrosis and by an inflammatory infiltration of lymphocytes. The duct epithelium is not stimulated to proliferate. This absence of epithelial proliferation is one of the basic features of mammary duct ectasia [1]. In due course fibrosis produces skin and nipple retraction. Squamous metaplasia of nipple ducts is normally absent [9].

In the intermediate stages of the disease, dilated ducts contain soft, yellowish-green material [2]. In contrast to the inflammation seen in granulomatous mastitis, in duct ectasia the inflammation is entirely periductal and confined to the large subareolar ducts. Initially, lymphocytes and plasma cells surround dilated ducts containing amorphous debris and foamy histiocytes. The lining epithelium is more commonly attenuated and may be replaced entirely by granulation tissue, often containing foreign-body giant cells and foamy macrophages. As the disease advances, progressive periductal fibrosis develops, with eventual obliteration of the duct lumen [2].

Atrophy rather than proliferation of the epithelium of the involved ducts is the rule. The epithelium is often so thinned out that it is barely visible in low power magnification as a thin dark line lying upon the dense collar of fibrous tissue that forms the bulk of the much thickened duct wall. Outside the fibrous collar there is usually a zone of lymphocytic infiltration demarcating the duct from surrounding fatty breast stroma. The matrix within the ducts contains amorphous debris with characteristic crystalline bodies scattered throughout it. As the mammary duct ectasia progresses, continuity of the atrophic duct epithelium is broken in places [1]. 
Although a rare situation in childhood, duct ectasia may be manifested as a bloody mammillary discharge [7]. Another situation in which duct ectasia is rare is in men. Discharge and mammillary retraction are the forms of clinical presentation, although the presence of an areolar nodule could occur [8].

On radiologic examination, calcification may be seen and the lesion mimics duct ectasia or, rarely, ductal carcinoma in-situ [2]. Mammogram shows tubular, annular and linear shadows due to the presence of calcifications. There is usually no accompanying epithelial hyperplasia or apocrine metaplasia [3]. The mammographic findings can be asymmetric which can simulate a carcinoma [10]. Ultrasonography, which is an easily reproducible, noninvasive examination of low cost, allows duct diameters greater than $5 \mathrm{~mm}$ to be diagnosed and measured $[11,12]$. The primary significance is that it produces an ill defined palpable mass that mimics the mammographic appearance of carcinoma [9].

Mammary duct ectasia is probably unrelated to fibrocystic disease, although the two may coexist. For the record, the condition is not too uncommon in female dogs, whether sexually intact or spayed [13].

Mammary duct ectasia is a lesion of the inactive and aging breast. The approach towards duct ectasia is usually conservative. Surgical options are microdochectomy or cone excision of major ducts. Surgery is reserved for cases associated with suspected malignancies.

\section{Case Report}

A 24-year-old unmarried female patient, resident of Kerala, presented with painful axillary swelling of 3 months duration. Family history was unremarkable. On physical examination the patient was well oriented and conscious with vital parameters within normal limits. Other biochemical and hematological parameters were within normal limits. The patient had come to the OP for cosmetic removal and so surgical excision of the mass was advised. The excised specimen of axillary area was sent to the Department of Pathology for histopathological examination.

\subsection{Gross: Received specimen in 2 containers}

Container 1 labelled as B 12/14 A (Right axillary lump): Received an irregular fibrofatty tissue, partially covered by skin, whole measuring $5 \times 5.5 \times 1 \mathrm{~cm} . \mathrm{C} / \mathrm{S}$ - yellowish, lobulated appearance with irregular grey white areas having tiny cystic spaces filled with creamy material.

Container 2 labelled as B 12/14 B (Left axillary lump): Received an irregular fibrofatty tissue partially covered by skin, whole measuring $3.5 \times 4 \times 1.5 \mathrm{~cm}$. C/S - yellowish, lobulated appearance separated by tiny cystic spaces filled with creamy material.
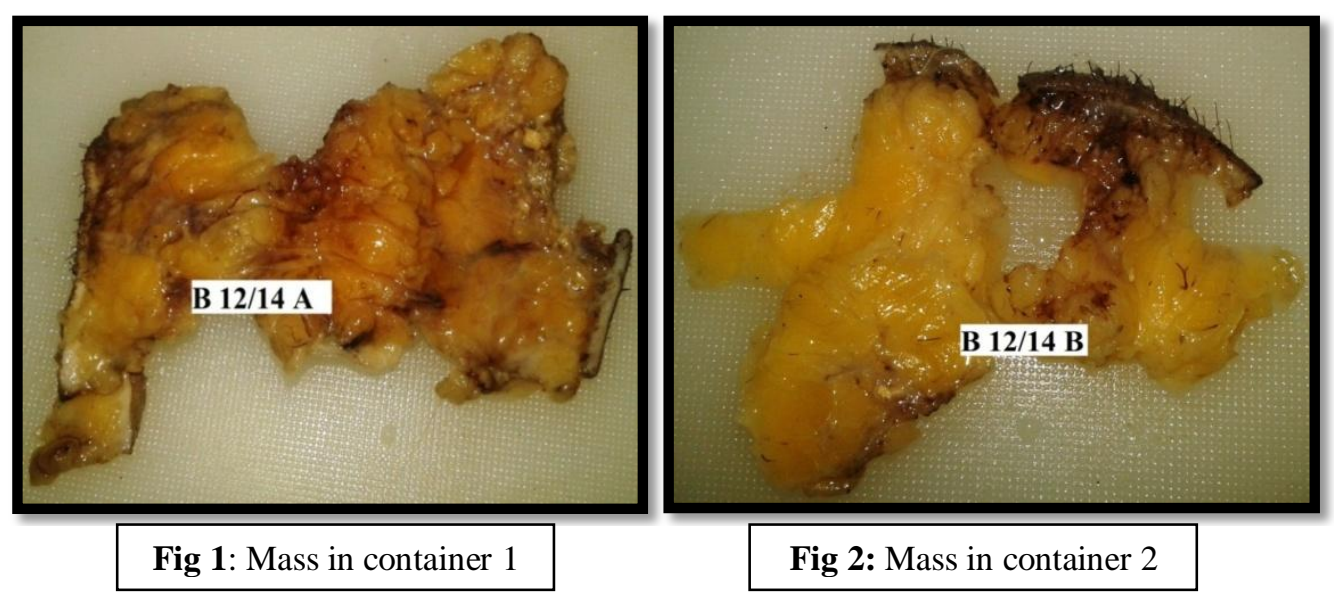

2.2 Microscopy: A \& B - Sections show tissue covered with intact skin. Dermis show dermal appendages. Subcutaneous fat shows breast tissue with few lobules of ducts lined by epithelial and myoepitheial cells. Some of the ducts are cystically dilated and filled with granular eosinophilic material lined by flattened cells. Stroma and periductal area show dense infiltration by mononuclears. Multiple bits and sections studied show similar appearance. 
2.3 Impression: A \& B - Excised axillary lumps- show breast tissue showing evidence of duct ectasia.

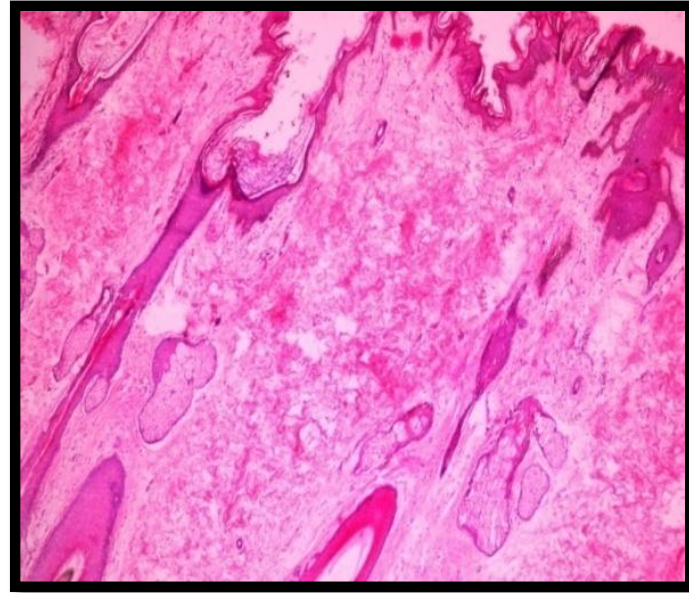

Fig 3: Photomicrograph showing tissue covered with intact skin with underlying skin adnexal structures $(4 \mathrm{x})$

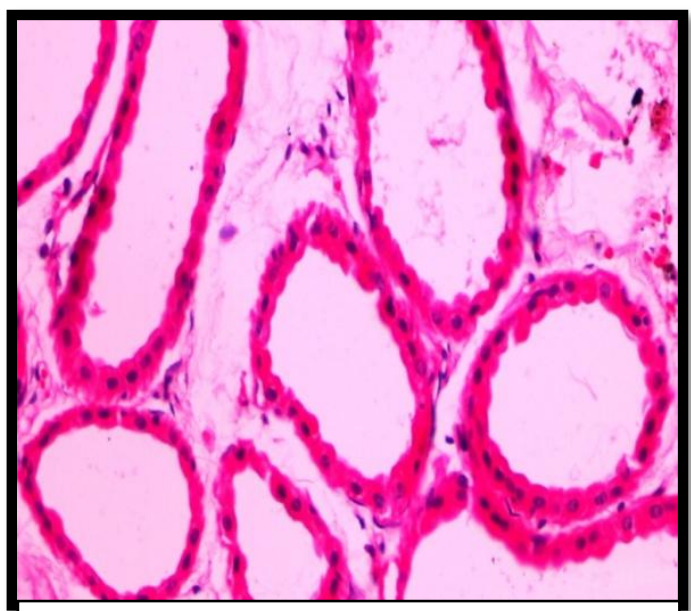

Fig 5: Photomicrograph showing ducts lined by epithelial and myoepithelial cells and showing apocrine change (40x)

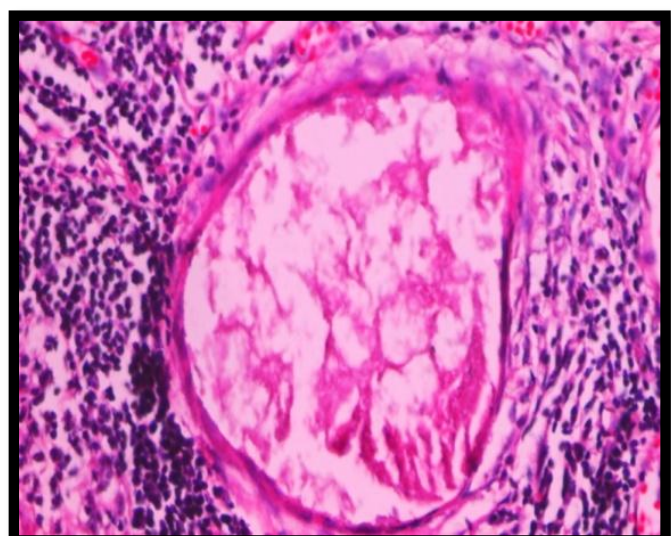

Fig 7: Photomicrograph showing cystically dilated duct lined by flattened cells and filled with granular eosinophilic material. Periductal area showing dense infiltration by mononuclear cells (40x)

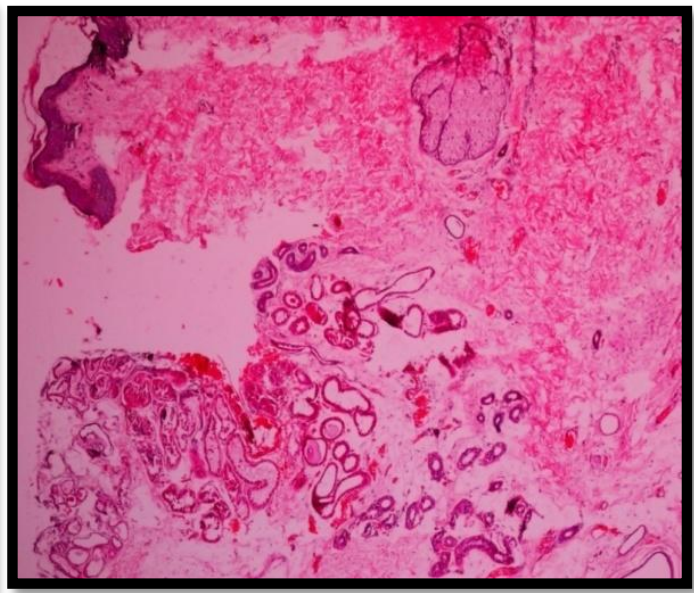

Fig 4: Photomicrograph showing tissue covered with intact skin with underlying skin adnexal structures and subcutaneous fat showing breast tissue with few lobules of ducts $(4 \mathrm{x})$
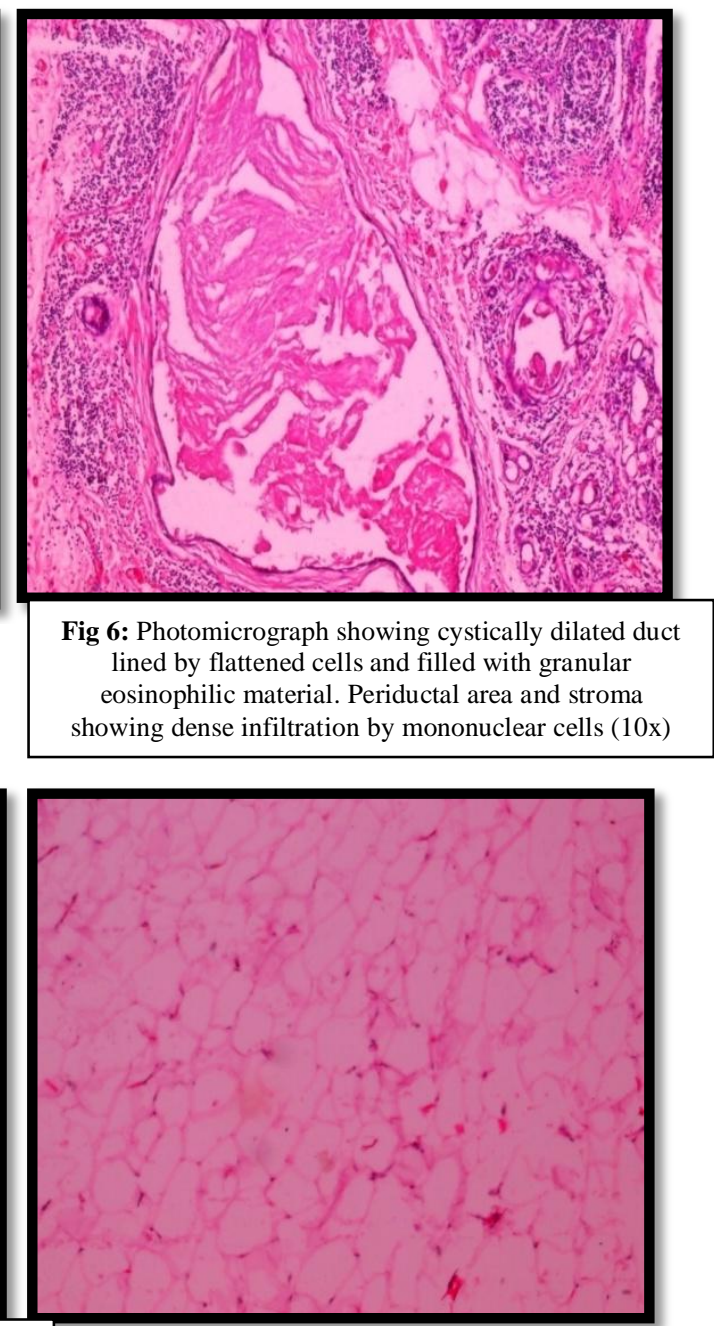

Fig 8: Photomicrograph showing normal appearing subcutaneous fat 


\section{Discussion}

On USG the swelling was reported as hypertrophied tail of breast. The gross examination showed only fibrofatty tissue with few areas of fibrosis. The definitive diagnosis could only be given by microscopic examination and the diagnosis of accessory breast with duct ectasia was given. Surgical excision is the treatment of choice as it relieves discomfort. Thus, this case gains significance because of the rarity of coexistence of accessory breast with duct ectasia without any primary lesion in the breast.

This case report is presented here because of its rarity as the age of the patient was only 24 yrs (normal age of presentation is pre or post menopausal women) and the patient did not have any nipple discharge/breast lesion (normal presenting complaint is nipple discharge with mass in unilateral/ bilateral breast).

\section{References}

[1]. Haagensen CD. Mammary duct ectasia - a disease that may simulate cancer. Cancer 4:749-761, 1951. Azzopardi JG, Cystic disease: duct ectasia: fat necrosis, in Bennington JL (Ed.), Problems in Breast Pathology, (Philadelphia: WB Saunders, 1979) 57-89.

[2]. Webb AJ. Mammary duct ectasia - periductal mastitis complex. Br J Surg 82:1300-1302, 1995.

[3]. Bartsich, Sophie A. M.D. Accessory Breast Tissue in the Axilla: Classification and Treatment. Plastic and reconstructive surgery 128 (1):35-36 2011.

[4]. O'Brien PH, Kreutner A Jr. Another cause of nipple discharge. Mammary duct ectasia with periductal mastitis. Am J Surg 48:577-578, 1982.

[5]. Rahul RM, de Freitas-Junior R, Paulinelli RR. Risk factors for duct ectasia. Breast J 11:262-265, 2005.

[6]. Kelly VM, Arif K, Ralston S, Gregor N, Scott S. Bloody nipple discharge in an infant and a proposed diagnostic approach. Pediatrics 117:814-816, 2006.

[7]. Chan KW, Lau WY. Duct ectasia in the male breast. Aust NZ J Surg 54:173-176, 1984

[8]. Meguid M, et al: Pathogenesis-based treatment of recurring subareolar breast abscesses. Surgery 118:775-779, 1995.

[9]. Browning J, Bigrigg A, Taylor I. Symptomatic and incidental mammary duct ectasia. J R Soc Med 79:715-716, 1986.

[10]. Sweeney DJ, Wylie EJ. Mammographic appearances of mammary duct ectasia that mimic carcinoma in a screening programme. Australas Radiol 39:18-23, 1995.

[11]. Rizzatto G, Chersevani R. Breast ultrasound and new technologies. Eur J Radiol 27 (2): 242-249, 1998.

[12]. Sakorafas GH. Nipple discharge: current diagnostic and therapeutic approaches. Cancer Treat Rev 27:275-282, 2001

[13]. Miller MA, Kottler SJ, Cohn LA, Johnson GC, Kreeger JM, Pace LV, Ramos-Vara JA, Turk JR, Turnquist SE. Mammary duct ectasia in dogs: 51 cases. J Am Vet Med Assoc 218:1303-1307, 2001. 